\title{
Artificial Bee Colony Algorithm with Adaptive Explorations and Exploitations: A Novel Approach for Continuous Optimization
}

\author{
Mohammad Shafiul Alam \\ Department of Computer \\ Science and Engineering \\ Ahsanullah University of \\ Science and Technology \\ Dhaka-1208, Bangladesh
}

\author{
Md. Monirul Islam \\ Department of Computer \\ Science and Engineering \\ Bangladesh University of \\ Engineering and Technology \\ Dhaka-1000, Bangladesh
}

\author{
Kazuyuki Murase \\ Department of Human and \\ Artificial Intelligence Systems \\ University of Fukui \\ Fukui 910-8507, Japan
}

\begin{abstract}
A proper balance between global explorations and local exploitations is often considered necessary for complex, high dimensional optimization problems to avoid local optima and to find a good near optimum solution with sufficient convergence speed. This paper introduces Artificial Bee Colony algorithm with Adaptive eXplorations and eXploitations $\left(\mathrm{ABC}-\mathrm{AX}^{2}\right.$ ), a novel algorithm that improves over the basic Artificial Bee Colony (ABC) algorithm. $\mathrm{ABC}-\mathrm{AX}^{2}$ augments each candidate solution with three control parameters that control the perturbation rate, magnitude of perturbations and proportion of explorative and exploitative perturbations. Together, all the control parameters try to adapt the degree of global explorations and local exploitations around each candidate solution by affecting how new trial solutions are produced from the existing ones. The control parameters are automatically adapted at the individual solution level, separately for each candidate solution. $\mathrm{ABC}-\mathrm{AX}^{2}$ is tested on a number of benchmark problems of continuous optimization and compared with the basic $\mathrm{ABC}$ algorithm and several other recent variants of $\mathrm{ABC}$ algorithm. Results show that the performance of $\mathrm{ABC}-\mathrm{AX} \mathrm{X}^{2}$ is often better than most other algorithms in comparison, in terms of both convergence speed and final solution quality.
\end{abstract}

\section{Keywords}

Artificial bee colony algorithm; Exploration and exploitation; Continuous optimization; Meta-heuristic optimization.

\section{INTRODUCTION}

The Artificial Bee Colony (ABC) algorithm is a recently introduced [1] swarm intelligence algorithm that tries to mimic the intelligent food foraging behavior of honey bees. Since its advent, the ABC algorithm has been successfully applied to wide and diverse range of problems, such as continuous optimization [2], discrete optimization [3], constrained optimization [4], multi-objective optimization [5], design optimization [6], training neural network [7], design of digital IIR filter [8], PID controller [9], parameterizing of milling processes [10] and so on [11]. ABC is simple in concept, easy to implement and requires fewer control parameters [12]. ABC shows very competitive and often better performance in comparison to many other existing evolutionary and swarm intelligence algorithms [2], such as genetic algorithm (GA), differential evolution (DE) and particle swarm optimization (PSO).
Similar to other population based meta-heuristic algorithms, $\mathrm{ABC}$ also has its own challenges and limitations. For example, $\mathrm{ABC}$ can prematurely converge to local optima, especially for complex high dimensional multimodal problems [2,13]. Also, the convergence speed of $\mathrm{ABC}$ is usually slower than some other meta-heuristic algorithms, such as DE and PSO, especially on unimodal problems [2]. Another problem that may occur with $\mathrm{ABC}$ is fitness stagnation [14], where the entire population of solutions stops improving, even without converging to some local optima, because the fitness based selection scheme fails to find new, better trial solutions that can enter the population by replacing the existing solutions. All these problems originate from a lack of balance between global explorations and local exploitations during the optimization procedure. $\mathrm{ABC}$ drives its search towards global optimum with two operators perturbation and selection. The perturbation operation is responsible for explorations by random variations of existing solutions, while the fitness based selection operation performs exploitations of the search regions explored so far. However, both these operations are more aligned towards exploitations than explorations. The perturbation operation of $\mathrm{ABC}$ perturbs a single parameter of an existing solution and thus produces the new trial solution in the neighborhood of the original solution, which is exploitative. The selection operation of $\mathrm{ABC}$ can accept only the better solutions, which is exploitative too. This paper introduces $\mathrm{ABC}$ with Adaptive eXplorations and eXploitations $\left(\mathrm{ABC}-\mathrm{AX}^{2}\right)$, a novel improvement over the basic $\mathrm{ABC}$ algorithm that tries to automatically adapt the degree of explorations and exploitations, separately for every candidate solution of the population. $\mathrm{ABC} \mathrm{AX}^{2}$ augments each candidate solution $\boldsymbol{x}_{i}$ with three control parameters $-p_{i}, q_{i}$ and $\boldsymbol{\eta}_{i}$, each of which affects the perturbation operations on $x i$ to control the degree of explorations and exploitations around $\boldsymbol{x}_{i}$. The values of $p_{i}$, $q_{i}$ and $\boldsymbol{\eta}_{i}$ are automatically adjusted, cycle (i.e., iteration) by cycle, using adaptive and self-adaptive techniques to increase the likelihood of producing more effective perturbations on $\boldsymbol{x}_{i}$. $\mathrm{ABC}-\mathrm{AX}^{2}$ is tested on a benchmark suite of 30 continuous functions of different complexity. Results are compared with the basic $\mathrm{ABC}$ [2] and several other recent variants of $\mathrm{ABC}$ (e.g., [15]-[21]), which show that $\mathrm{ABC}-\mathrm{AX}^{2}$ often performs better than most other $\mathrm{ABC}$-variants in comparison. 


\section{THE ABC ALGORITHM}

Honey bees in nature have to forage over a vast area in search of good sources of nectar. After an initial exploration stage, more bees are employed to collect honey from more profitable food sources whereas fewer bees are assigned to the less worthy sources. After returning the hive, each bee goes to the 'dance floor' and performs a special dance known as the 'waggle dance' to share the information of the food source it has found. The 'onlooker' bees, waiting around the dance floor, observe the waggle dances of the 'employed' bees and pick any of them to follow and collect nectar from the vicinity of its food source. Some scout bees are also assigned for random explorations of the search space to find new food sources. The basic ABC algorithm [1,2] mimics the food foraging behavior of honey bees with the same three groups of bees - employed, onlooker and scout bees. A bee working to forage a particular food source (i.e., candidate solution) and searching only around its vicinity is called an employed bee. Onlooker bees randomly pick and follow any of the employed bees. The probability of picking an employed bee is proportional to the quality of its food source. Scout bees can perform random explorations of the search space to find new food sources. If the employed and onlooker bees, even after limit attempts, fail to find a better food position around a particular food $\boldsymbol{x}_{i}$, then $\boldsymbol{x}_{i}$ is abandoned and replaced by initiating a scout bee and its food source is placed uniformly at random across the search space. In the original implementation of the $\mathrm{ABC}$ algorithm, half of the colony is employed bees, the other half is onlooker bees, and scout bees are created on demand only when a food source fails to improve with several attempts. Fig. 1 presents the pseudocode for the basic $\mathrm{ABC}$ algorithm. Each cycle (i.e., iteration) of $\mathrm{ABC}$ consists of foraging by the employed bees (steps 4-5, Fig. 1), then foraging by the onlookers (steps 7-9), followed by placement of the scout bees (step 10). Each of these stages is described below.

Foraging by employed bees: Suppose, an employed bee is currently positioned at a food source position $\boldsymbol{x}_{i}$. During this stage, each employed bee searches in the vicinity of its current position $\boldsymbol{x}_{i}$ to produce new trial food source $\boldsymbol{v}_{i}$ using (1), where $j \in\{1,2, \ldots, D\}$ and $k \in\{1,2, \ldots, S N\}$ are randomly picked indices, $D$ is dimensionality of the problem, $S N$ is the number of food positions and $\varphi_{i j}$ is a uniform random value $\sim[-1,1]$.

$$
v_{i j}=x_{i j}+\varphi_{i j}\left(x_{k j}-x_{i j}\right)
$$

Thus, the new solution $\boldsymbol{v}_{i}$ is produced from $\boldsymbol{x}_{i}$ by perturbing its randomly picked $j$-th parameter and using the information of $\boldsymbol{x}_{i}$ and another randomly picked solution $\boldsymbol{x}_{k}$. If $\boldsymbol{v}_{i}$ has better 'fitness' than the old food position $\boldsymbol{x}_{i}$, then $\boldsymbol{x}_{i}$ is replaced by $\boldsymbol{v}_{i}$. For the problem of function optimization, where $f$ is the function to be minimized, ABC computes the 'fitness' of a candidate solution $\boldsymbol{x}_{i}$ using (2).

$$
\text { fitness }\left(\boldsymbol{x}_{i}\right)= \begin{cases}\frac{1}{1+f\left(\boldsymbol{x}_{i}\right)} ; & \text { if } f\left(\boldsymbol{x}_{i}\right) \geq 0 \\ 1+\left|f\left(\boldsymbol{x}_{i}\right)\right| & \text { otherwise }\end{cases}
$$

Foraging by onlooker bees: During this stage, each onlooker bee randomly picks an employed bee to follow and forages only around the vicinity of its food source. The probability $w_{i}$ that the employed bee with food source $\boldsymbol{x}_{i}$ would be picked by an onlooker bee is computed using (3), which makes the probability $w_{i}$ to be proportional to fitness $\left(\boldsymbol{x}_{i}\right)$.

$$
w_{i}=\frac{\operatorname{fitness}\left(x_{i}\right)}{\sum_{n=1}^{S N} \operatorname{fitness}\left(x_{n}\right)}
$$

Like the employed bees, each onlooker bee also employs (1) to produce trial food source $v_{i}$ in the vicinity of its current food source position $\boldsymbol{x}_{i}$. If $\boldsymbol{v}_{i}$ has better fitness than $\boldsymbol{x}_{i}$, then $\boldsymbol{x}_{i}$ is replaced by $\boldsymbol{v}_{i}$. Otherwise, $\boldsymbol{v}_{i}$ is discarded.

Placement of Scout bees: A scout bee is created only when a particular food source $\boldsymbol{x}_{\boldsymbol{i}}$ failed to be improved over the last 'limit' iterations. The bee employed to $\boldsymbol{x}_{\boldsymbol{i}}$ now becomes a scout bee and its food source is positioned at random across the search space using (4), where $j=1,2, \ldots, D$ and $\left[\min _{j}, \max _{j}\right]$ is the search space along the $j$-th dimension.

$$
x_{i j}=\min _{j}+\operatorname{rand}(0,1) *\left(\max _{j}-\min _{j}\right)
$$

\section{EXISTING VARIANTS OF ABC ALGORITHM}

There exist a number of recent studies (e.g., [15]-[24]) that try to alter the explorative and/or exploitative properties of the basic $\mathrm{ABC}$ algorithm. For example, $\mathrm{ABC}$ with self-adaptive mutation (ABC-SAM) [15] introduces an adaptive mutation scaling factor $S F_{i}$ for every candidate solution $\boldsymbol{x}_{i}$ and tries to ensure both explorations and exploitations by periodically adjusting the value of $S F_{i}$ using two different distributions one explorative and the other exploitative. The $S F_{i}$ values can be randomly re initialized, if necessary, to perform more explorations. The cooperative $\mathrm{ABC}$ (CABC) [16] tries to enforce more explorations by decomposing the search space into multiple subspaces and by employing multiple bee colonies to explore through different subspaces. $\mathrm{ABC}$ with diversity strategy (DABC) [17] tries to maintain sufficient level of population diversity for conducting more explorations by alternating between two different perturbation schemes. Chaotic $\mathrm{ABC}$ (ChABC) [18] tries to improve the explorative characteristics of $\mathrm{ABC}$ by employing chaotic dynamics instead of random number generators. The Gbest-guided $\mathrm{ABC}(\mathrm{GABC})[20]$ tries to improve the exploitations and convergence speed of $\mathrm{ABC}$ by altering its perturbation operation using the information of the global best solution found so far. Hooke Jeeves ABC (HJABC) [21-22] is a hybrid $\mathrm{ABC}$-variant that intensifies the exploitative operations by hybridizing $\mathrm{ABC}$ with a local search technique (i.e., the Hooke Jeeves pattern search). The Elitist ABC (EABC) [24] is another exploitative $\mathrm{ABC}$ variant that hybridizes $\mathrm{ABC}$ with two different local search operators to intensify the degree of exploitations around the best candidate solution found so far. Thus, most existing ABC-variants try to improve either the exploitative (e.g., [20]-[24]) or the explorative ([15]-[19]) characteristics of the basic $\mathrm{ABC}$ algorithm. The exploitative improvements are usually based on intensifying the search around the best solution(s) found so far (e.g., [20], [21], [24]) and hybridizing efficient local search operators with $\mathrm{ABC}$ (e.g., [21], [23], [24]), while the explorative improvements can be based on more population diversity (e.g., [16], [17]) and more explorative selection and/or perturbation operations ([15], [18], [19]). But none of these algorithms considers the individual explorative/exploitative requirements of each 
candidate solution separately; rather they employ some population-wide global strategy, identically for all candidate solutions, which is significantly improved in the proposed algorithm $-\mathrm{ABC}-\mathrm{AX}^{2}$, as described in the following section.

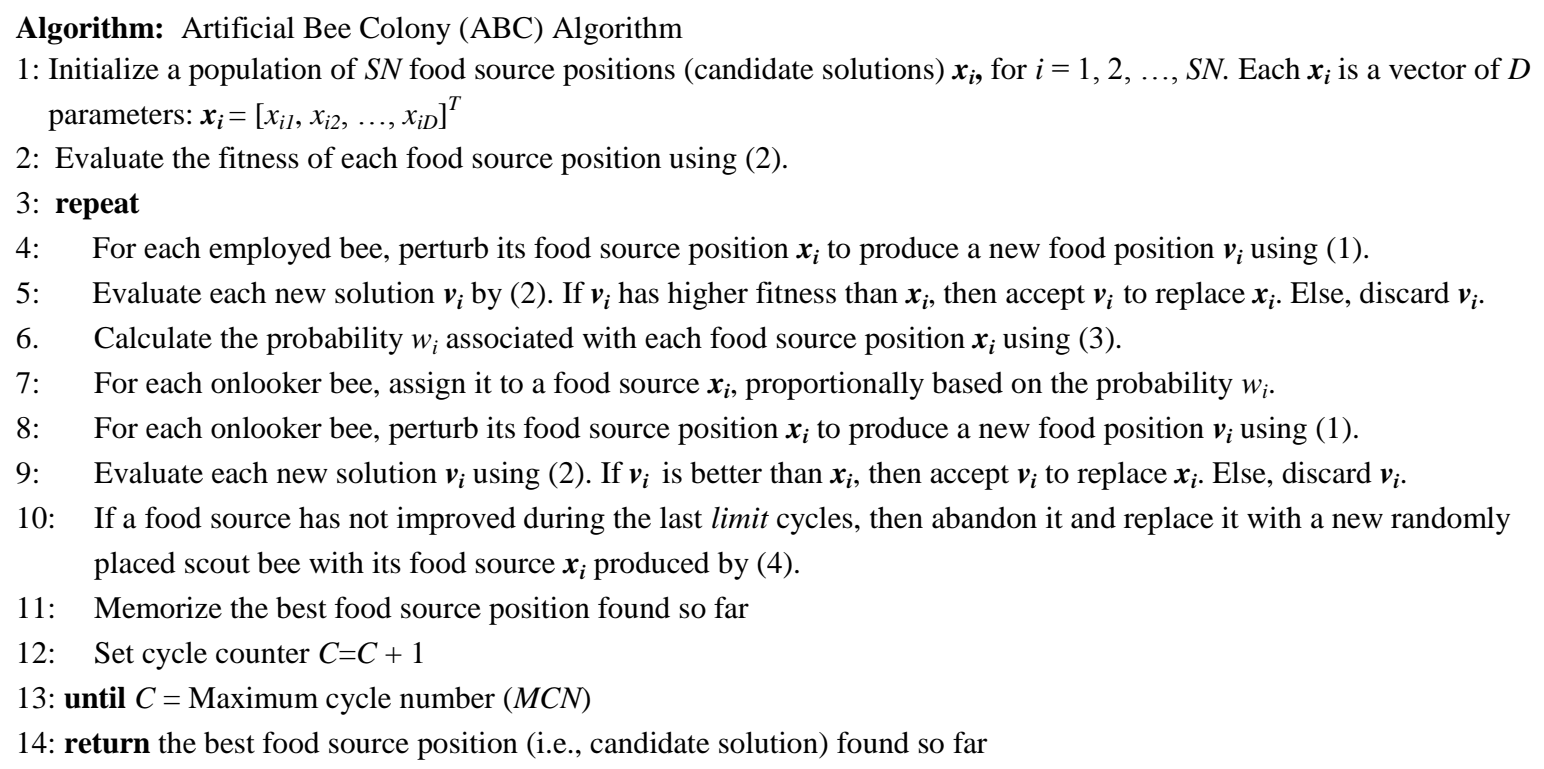

Fig. 1: Algorithm for the basic Artificial Bee Colony (ABC) algorithm

\section{THE PROPOSED ALGORITHM - ABC-AX ${ }^{2}$}

$\mathrm{ABC}-\mathrm{AX}^{2}$ tries to improve over the basic $\mathrm{ABC}$ algorithm by adapting and customizing the degree of explorations and exploitations at the individual solution level, i.e., separately for every candidate solution. $\mathrm{ABC}-\mathrm{AX}^{2}$ includes three control parameters $-p_{i}, q_{i}$ and $\boldsymbol{\eta}_{i}$ within each candidate solution $\boldsymbol{x}_{\boldsymbol{i}}$. The control parameter $p_{i}$ controls the proportion of explorative and exploitative perturbations; $q_{i}$ controls the perturbation rate to produce $\boldsymbol{v}_{\boldsymbol{i}}$ from $\boldsymbol{x}_{\boldsymbol{i}} ; \boldsymbol{\eta}_{\boldsymbol{i}}=\left[\eta_{i 1}, \eta_{i 2}, \ldots, \eta_{i D}\right]^{T}$ is a vector with $D$ components, each one (say, $\eta_{i j}$ ) of which controls the distribution of the scaling factor values (i.e., $\varphi_{i j}$ values in (1)) during perturbations along the corresponding (i.e., $j$-th) dimension. Each control parameter is gradually adapted to achieve higher rate of 'successful' perturbations. A perturbation is considered 'successful' only if the new trial solution $\boldsymbol{v}_{\boldsymbol{i}}$ has higher fitness value than the original solution $\boldsymbol{x}_{\boldsymbol{i}}$. A detailed description of the role of each control parameter, how it affects explorations and exploitations in perturbations and how it is gradually adapted by $\mathrm{ABC}-\mathrm{AX}^{2}$ are presented in the following paragraphs.

A. Control parameter $p_{i}$ for adaptive proportion of explorations and exploitations: The basic $\mathrm{ABC}$ algorithm uses the single perturbation scheme (1), with no attempt to differentiate between explorative or exploitative perturbations. In contrast, $\mathrm{ABC}-\mathrm{AX}^{2}$ employs two different perturbation schemes - one for explorations, the other for exploitations. Both the perturbation schemes are based on the same expression (1), but they differ in how $\boldsymbol{x}_{i}$ selects its supporting candidate solution $\boldsymbol{x}_{k}$ in (1). For explorative perturbations, $\boldsymbol{x}_{k}$ is picked by three-tier explorative tournament selection (3T-ER-TS), while the exploitative perturbations use two-tier exploitative tournament selection (2T-ET-TS) procedure. Both the selection procedures are introduced in Fig. 2.

\section{Explorative perturbation:}

$$
v_{i j}=x_{i j}+\varphi_{i j}\left(x_{k j}-x_{i j}\right), \text { where } \boldsymbol{x}_{k} \sim 3 \mathrm{~T}-\mathrm{ER}-\mathrm{TS}\left(\boldsymbol{x}_{i}\right)
$$

\section{Exploitative perturbation:}

$$
v_{i j}=x_{i j}+\varphi_{i j}\left(x_{k j}-x_{i j}\right), \text { where } \boldsymbol{x}_{k} \sim 2 \mathrm{~T} \text {-ET-TS }\left(\boldsymbol{x}_{i}\right)
$$

The explorative 3T-ER-TS scheme tries to pick a candidate solution $\boldsymbol{x}_{k}$ that is not only fit, but also dissimilar (from the current solution $\boldsymbol{x}_{i}$ ) and diverse (from the other solutions of the population). Dissimilarity of $\boldsymbol{x}_{k}$ from $\boldsymbol{x}_{i}$ is measured as their Euclidean distance (ED), while diversity of $\boldsymbol{x}_{k}$ is estimated as its ED from the centroid of population of solutions. High dissimilarity of $\boldsymbol{x}_{k}$ from $\boldsymbol{x}_{i}$ ensures a large $\left|x_{k j}-x_{i j}\right|$ in (5) to make a large, explorative perturbation on $\boldsymbol{x}_{i}$, while the high diversity of $\boldsymbol{x}_{k}$ tries to pull $\boldsymbol{x}_{i}$ away from the population centroid to promote more diversity and to avoid being trapped around local optima. In contrast, the exploitative 2T-ET-TS scheme tries to pick an $\boldsymbol{x}_{k}$ that is both fit and has high degree of similarity to $\boldsymbol{x}_{\boldsymbol{i}}$. This tries to ensure a small $\left|x_{k j}-x_{i j}\right|$ in (6) to make small, exploitative steps towards the better regions of the search space.

But how does $\mathrm{ABC}-\mathrm{AX}^{2}$ decide on whether to perform explorative or exploitative perturbation on $\boldsymbol{x}_{i}$ ? This is done probabilistically - the current values of $p_{i}$ and $1-p_{i}$ denote the probability of exploitative and explorative perturbations on $\boldsymbol{x}_{i}$, respectively. The value of $p_{i}$ is automatically adapted using the incremental learning experience of $\boldsymbol{x}_{i}$, which includes the number of successes and failures by explorative and exploitative perturbations on $\boldsymbol{x}_{i}$ during the last $\tau_{1}$ cycles (learning period). Initially, $p_{i}$ is set to 0.5 for every solution $\boldsymbol{x}_{i}$, 
which makes exploitative and explorative perturbations equally desired. After the initial learning period of $\tau_{1}$ cycles, $\mathrm{ABC}-\mathrm{AX}^{2}$ starts adjusting the $p_{i}$ value for each $\boldsymbol{x}_{i}$. To do this, $\mathrm{ABC}-\mathrm{AX}^{2}$ keeps record of the number of successes and failures by exploitative and explorative perturbations on $\boldsymbol{x}_{i}$ over the last $\tau_{1}$ cycles. Suppose $n s_{E R}$ and $n f_{E R}\left(n s_{E T}\right.$ and $\left.n f_{E T}\right)$ are the number of successes and failures, respectively by the explorative (exploitative) perturbations on $\boldsymbol{x}_{i}$ during the last $\tau_{1}$ cycles. Then, success ratios of explorative perturbation $\left(S R_{E R}\right)$ and exploitative perturbation $\left(S R_{E T}\right)$ on $\boldsymbol{x}_{i}$ are computed as: $S R_{E R}=\left(n s_{E R}\right) /\left(n s_{E R}+n f_{E R}\right)$ and $S R_{E T}=\left(n s_{E T}\right) /\left(n s_{E T}+n f_{E T}\right)$. Now, the adjusted probability of exploitative perturbation on $\boldsymbol{x}_{i}$ (i.e., the adjusted value of $p_{i}$ ) is computed using (7), which also ensures $0.1 \leq p_{i} \leq 0.9$ to avoid the complete domination by either mode of perturbations. Once the value of $p_{i}$ for each candidate solution $\boldsymbol{x}_{i}$ is computed by $\mathrm{ABC}-\mathrm{AX}^{2}$ using (7), it is kept unchanged for the next $\tau_{2}$ cycles $\left(\tau_{2}<\tau_{1}\right)$, which allows some time for the adjusted value of $p_{i}$ to produce both successes and failures by each type of perturbation. ABC-AX ${ }^{2}$ regularly adjusts the value of $p_{i}$ for each candidate solution $\boldsymbol{x}_{i}$ using (7), periodically after each $\tau_{2}$ cycles, using the recorded values of number of successes and failures by each type of perturbation on $\boldsymbol{x}_{i}$ over the last $\tau_{1}$ cycles. After some initial experiments, these parameters are set as $\tau_{1}=50$ and $\tau_{2}=10$.

$$
p_{i}=\min \left(0.9, \max \left(0.1, \frac{S R_{E T}}{S R_{E R}+S R_{E T}}\right)\right)
$$

B. Control parameter $q_{i}$ for self-adaptive perturbation rate: The basic $\mathrm{ABC}$ algorithm perturbs only a single, random parameter of $\boldsymbol{x}_{i}$ using (1). This usually produces the trial solution $\boldsymbol{v}_{i}$ in the neighbourhood of the original solution $\boldsymbol{x}_{i}$, which is exploitative. Perturbing a single parameter allows search along a single dimension at a time. This may work well for separable problems, but not suitable for non-separable problems where the parameters are not independent. Fig. 3 shows an example using a 2D search space. Allowing perturbation of both the parameters (i.e., $x_{i 1}$ and $x_{i 2}$ ) can produce $\boldsymbol{v}_{i}$ along any possible direction from $\boldsymbol{x}_{i}$. This is more efficient than perturbing either $x_{i 1}$ or $x_{i 2}$, one at a time, as is done by the basic $\mathrm{ABC}$ algorithm that allows search along axis directions only. In contrast, $\mathrm{ABC}-\mathrm{AX}^{2}$ tries to perform search along any possible direction from $\boldsymbol{x}_{i}$ by maintaining and automatically adapting a control parameter $q_{i}$, separately for every candidate solution $\boldsymbol{x}_{i}$, that controls the perturbation rate during producing the trial solution $\boldsymbol{v}_{i}$ from $\boldsymbol{x}_{i}$. When $\mathrm{ABC}-\mathrm{AX}^{2}$ wants to perturb a solution $\boldsymbol{x}_{i}$ to produce $\boldsymbol{v}_{i}$, the value of $q_{i}$ is perturbed first, with probability $=u_{1}$ using (8), before perturbing any other parameter of $\boldsymbol{x}_{i}$. This perturbed value of $q_{i}$ is inherited by $\boldsymbol{v}_{i}$, which is henceforth referred as $v_{i} \cdot q$ and is used as the probability of perturbing the parameters of $\boldsymbol{x}_{i}$ during producing $\boldsymbol{v}_{i}$ from $\boldsymbol{x}_{i}$. A more appropriate value of $\boldsymbol{v}_{i} \cdot q$ is likely to produce fitter new solutions, which are supposed to survive better than $\boldsymbol{x}_{i}$ and produce better, newer solutions and hence, propagate the better value of the perturbation probability. Thus a gradual self-adaptation towards better, more effective $q_{i}$ values takes place, allowing a self-adaptive and appropriate perturbation rate for the candidate solutions across the population.

$v_{i} \cdot q= \begin{cases}q_{\min }+\operatorname{rand}(0,1) *\left(q_{\max }-q_{\min }\right) & \text { if } \operatorname{rand}(0,1) \leq u_{1} \\ q_{i} & \text { otherwise }\end{cases}$
Here, $u_{1}$ is the probability that the perturbation probability $q_{i}$ itself is perturbed before perturbing the parameters of $\boldsymbol{x}_{\boldsymbol{i}}$. In $\mathrm{ABC}-\mathrm{AX}^{2}$ implementation, these parameters have been set as $u_{1}=0.10, q_{\max }=1.0$ and $q_{\min }=1 / D$.

C. Control parameter $\eta_{i}$ for self-adaptive perturbation scaling factors: The basic $\mathrm{ABC}$ algorithm draws the $\varphi_{i j}$ values in (1) uniformly at random from $[-1,1]$, without any attempt to perform adaptation of the $\varphi_{i j}$ values for more effective perturbations on $\boldsymbol{x}_{i}$. In contrast, $\mathrm{ABC}-\mathrm{AX}{ }^{2}$ produces $\varphi_{i j}$ values from a Gaussian distribution with mean $=0$ and standard deviation $=\eta_{i j}$, where $\boldsymbol{\eta}_{i}=\left[\eta_{i l}, \eta_{i 2}, \ldots, \eta_{i D}\right]^{T}$ is a control parameter vector that is maintained separately for each candidate solution $\boldsymbol{x}_{i}$ and is gradually self-adapted using (9) and (10). Although this procedure is similar to the self-adaptation strategy adopted in some other previous evolutionary algorithms [25], it has not yet been employed and tested with the $\mathrm{ABC}$ algorithm.

$$
\begin{aligned}
& \text { for } j= 1,2, \ldots, D \\
& \eta_{i j}^{\prime}=\eta_{i j} \exp \left(\tau^{\prime} N(0,1)+\tau N_{j}(0,1)\right) \\
& \boldsymbol{v}_{i . \boldsymbol{\eta}}= \begin{cases}\boldsymbol{\eta}_{\boldsymbol{i}}^{\prime} & \text { if } \operatorname{rand}(0,1) \leq u_{2} \\
\boldsymbol{\eta}_{\boldsymbol{i}} & \text { otherwise }\end{cases}
\end{aligned}
$$

Here $u_{2}$ is the probability that the new trial solution $\boldsymbol{v}_{i}$ gets a control parameter $\boldsymbol{v}_{i} \cdot \boldsymbol{\eta}$ that is different from $\boldsymbol{\eta}_{i}$ of the original solution $\boldsymbol{x}_{i}$. ABC-AX ${ }^{2}$ uses $u_{2}=0.5$. The $N(0,1)$ and $N_{j}(0,1)$ are random numbers produced from the Normal distribution with mean $=0$ and standard deviation $=1$. The subscript $j$ in $N_{j}(0,1)$ indicates that the random number is generated anew for each value of $j$. The $\tau$ and $\tau^{\prime}$ are called learning rates and are set as suggested in [25]. ABC-AX ${ }^{2}$ maintains a separate $\boldsymbol{\eta}_{i}$ for every solution $\boldsymbol{x}_{i}$, which enables each $\boldsymbol{x}_{i}$ to customize its own degree of explorations and exploitations, separately along the $D$ different axis directions of the search space, using the components of $\boldsymbol{\eta}_{i}=\left[\eta_{i 1}, \eta_{i 2}, \ldots, \eta_{i D}\right]^{T}$. An effective value for $\boldsymbol{v}_{i} \cdot \boldsymbol{\eta}$ is likely to produce better, fitter new solutions that should survive better than $\boldsymbol{x}_{i}$ and thus a gradual self-adaptation towards better, more effective $\boldsymbol{\eta}_{i}$ values can take place, cycle by cycle, across the population.

\section{EXPERIMENTAL STUDIES}

To evaluate the performance of $\mathrm{ABC}-\mathrm{AX}^{2}$ and to compare it with the basic $A B C[2]$ and some other recent $A B C$-variants (e.g., [15]-[21]), this paper uses a set of benchmark problems which has 30 standard functions, including 18 scalable high dimensional functions with dimensionality $D=30,60$, as well as 12 low dimensional multimodal functions with $D \leq 10$. The suite contains both unimodal (i.e., $f_{1}-f_{9}$ ) and multimodal (i.e., $f_{10}-f_{30}$ ), separable (e.g., $f_{1}, f_{3}, f_{8}$ ) and non-separable (e.g., $f_{2}, f_{4}$, $f_{5}$ ), high (i.e., $f_{1}-f_{18}$ ) and low (i.e., $f_{19}-f_{30}$ ) dimensional functions. These functions have been widely used with many other evolutionary and swarm intelligence algorithms (e.g., [2], [15]-[18], [26]-[28]). Each function is briefly presented in Table 1. More details can be found in [2], [21], [28].

\subsection{ABC-AX ${ }^{2}$ on Standard Benchmark Functions}

Based on their properties, the benchmark functions (Table 1) can be divided into three groups - functions with no local minima (i.e., unimodal functions $f_{1}-f_{9}$ ), large number of local 
minima (i.e., high dimensional multimodal functions $f_{10}-f_{18}$ ) and only a few local minima (i.e., low dimensional multimodal functions $f_{19}-f_{30}$ ). To minimize a multimodal function, the optimization algorithm should have both explorative and exploitative capabilities, because it has to avoid being trapped around the locally minimal points and continue both explorations and exploitations until it locates the neighbourhood of a global minimum. Some of the multimodal functions can have tens or even hundreds of local minima, even with just two dimensions (e.g., Rastrigin

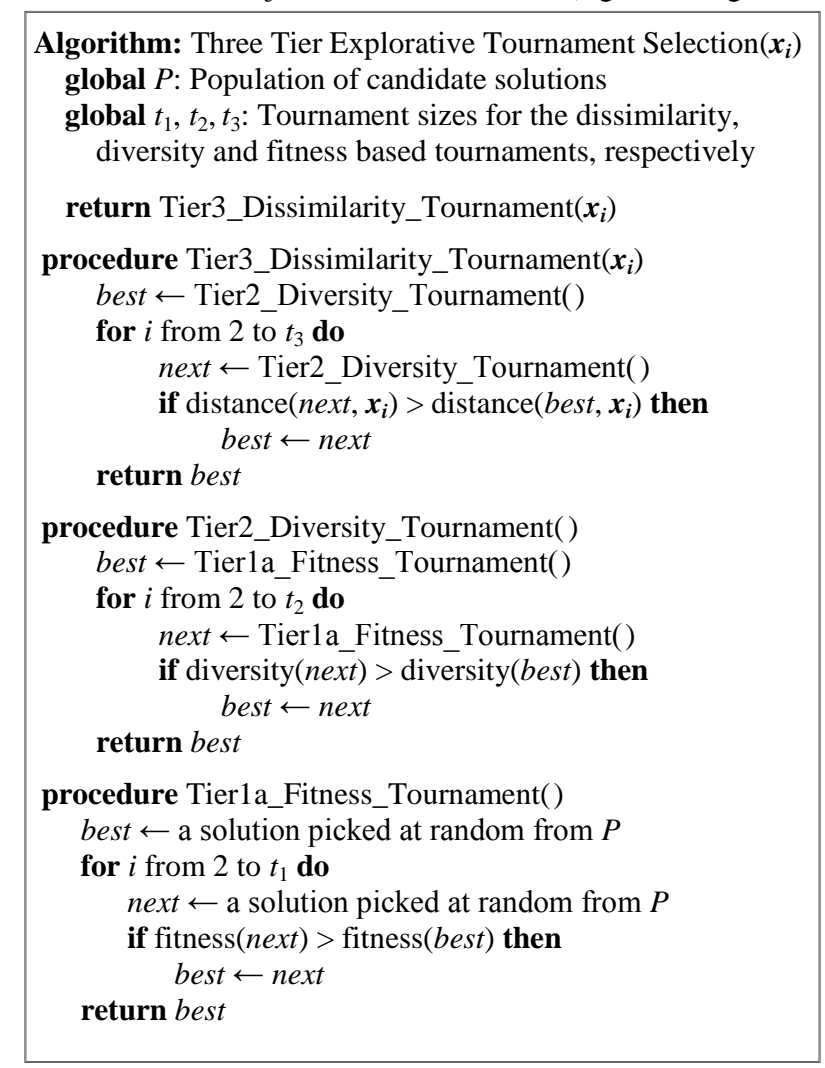

function $f_{10}$ ). The number of local minima can increase exponentially with the number of dimensions, which makes the optimization extremely difficult.

Table 2 presents the results of $\mathrm{ABC}-\mathrm{AX}^{2}$ on the 30 standard benchmark functions and compares the results with the basic $\mathrm{ABC}[2]$ and $\mathrm{ABC}$ with self-adaptive mutation (ABC-SAM) [15]. All the algorithms have made 50 independent runs on each function and the mean and standard deviation of the best found solutions are presented in Table 2.

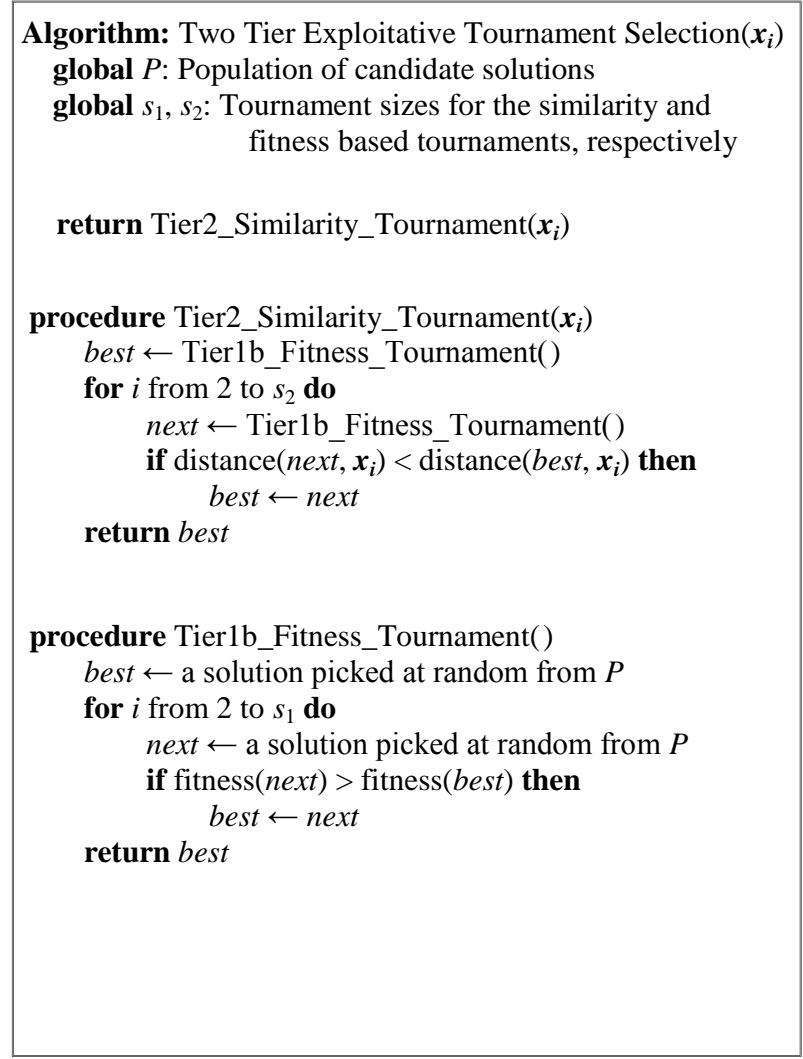

Fig. 2: Pseudocode for three-tier explorative tournament selection (on the left) and two-tier exploitative tournament selection (on the right) for $\mathrm{ABC}-\mathrm{AX}^{2}$
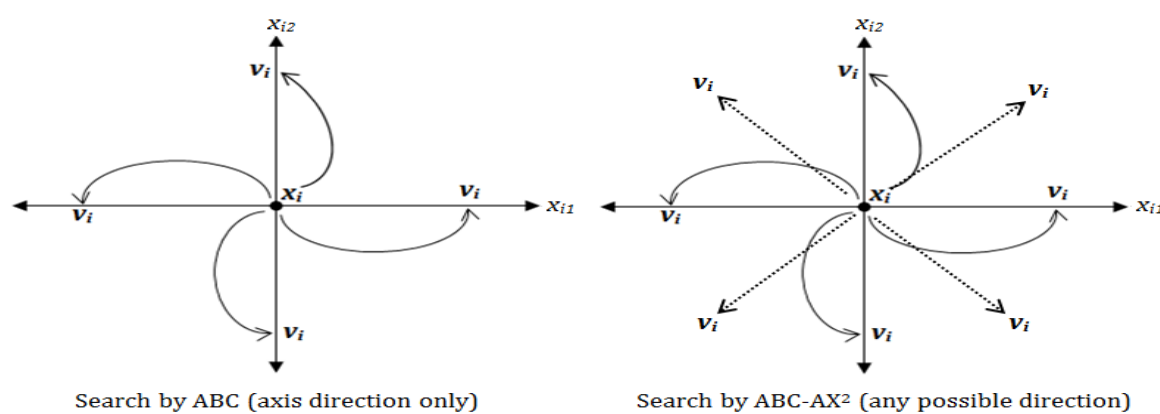

Fig. 3: Search direction by $\mathrm{ABC}$ (on the left) and $\mathrm{ABC}-\mathrm{AX}^{2}$ (on the right) in $2 \mathrm{D}$ search space

These algorithms have three parameters in common, which are population size $S N$, maximum cycle number $M C N$ and limit. For functions $f_{1}-f_{18}$ with $D=30, \mathrm{ABC}-\mathrm{AX}^{2}$ used $S N=100$, $M C N=1000$ and limit $=100$. For the larger variants with $D=60$, the value of $S N$ is kept the same (i.e., 100), but limit and $M C N$ are set to 200 and 2000, respectively. For the low dimensional $f_{19}-f_{30}, \mathrm{ABC}-\mathrm{AX}^{2}$ sets $S N=100, M C N=100$ and limit $=10 * D$. The other parameters of $\mathrm{ABC}-\mathrm{AX}^{2}$ are set as: $\tau_{1}=50, \tau_{2}=10$, $u_{1}=0.1, u_{2}=0.5, q_{\min }=1 / D, q_{\max }=1.0$. Tournament sizes for 3T-ETS and 2T-ETS selection schemes (Fig. 2) are set as: $t_{1}=t_{2}=6, t_{3}=4$, and $s_{1}=6, s_{2}=4$. During initializations, control parameter $p_{i}$ of each solution $\boldsymbol{x}_{i}$ is set to 0.5 , and the $q_{i}$ and $\eta_{i j}$ values are initialized to random values from $\left[q_{\min }, q_{\max }\right]$ and $[-1,1]$, respectively. These values are chosen with some initial experiments and not meant for optimum. The results in Table 2 are summarized in the following points. 
- $\mathbf{A B C}$ vs. $\mathbf{A B C}-\mathbf{A X}^{2}$ : Out of the 18 high dimensional functions $f_{1}-f_{18}, \mathrm{ABC}-\mathrm{AX}^{2}$ outperforms $\mathrm{ABC}$ on as many as 16 functions, shows similar performance on one $\left(f_{8}\right)$, while $\mathrm{ABC}$ manages to perform better only on one function $\left(f_{7}\right)$. Each time, the difference is statistically significant, as measured by $t$-test with $99 \%$ confidence interval. For the low dimensional functions $f_{19}-f_{30}$, both $\mathrm{ABC}$ and $\mathrm{ABC}-\mathrm{AX}^{2}$ perform equally well on eight functions, while $\mathrm{ABC}-\mathrm{AX}^{2}$ performs better on other four.

Table 1. Benchmark functions for experimental study. $D$ : dimensionality of the function, $S$ : search space, $f_{\text {min }}$ : function value at global minimum, $C$ : function characteristics with values $-U$ : Unimodal, $M$ : Multimodal, $S$ : Separable and $N$ : Non-Separable.

\begin{tabular}{|c|c|c|c|c|c|}
\hline No & Function & $D$ & $S$ & $C$ & $f_{\min }$ \\
\hline$f_{1}$ & Sphere & 30 and 60 & {$[-100,100]^{D}$} & $U S$ & 0 \\
\hline$f_{2}$ & Schwefel 2.22 & 30 and 60 & {$[-10,10]^{D}$} & $U N$ & 0 \\
\hline$f_{3}$ & Schwefel 2.21 & 30 and 60 & {$[-10,10]^{D}$} & $U S$ & 0 \\
\hline$f_{4}$ & Schwefel 1.2 & 30 and 60 & {$[-100,100]^{D}$} & $U N$ & 0 \\
\hline$f_{5}$ & Powell & 24 & {$[-4,5]^{D}$} & $U N$ & 0 \\
\hline$f_{6}$ & Dixon-Price & 30 and 60 & {$[-10,10]^{D}$} & $U N$ & 0 \\
\hline$f_{7}$ & Rosenbrock & 30 and 60 & {$[-30,30]^{D}$} & $U N$ & 0 \\
\hline$f_{8}$ & Step & 30 and 60 & {$[-100,100]^{D}$} & $U S$ & 0 \\
\hline$f_{9}$ & Quartic & 30 and 60 & {$[-1.28,1.28]^{D}$} & $U S$ & 0 \\
\hline$f_{10}$ & Rastrigin & 30 and 60 & {$[-5.12,5.12]^{D}$} & $M S$ & 0 \\
\hline$f_{11}$ & $\begin{array}{l}\text { Non-continuous } \\
\text { Rastrigin }\end{array}$ & 30 and 60 & {$[-5.12,5.12]^{D}$} & $M S$ & 0 \\
\hline$f_{12}$ & Schwefel & 30 and 60 & {$[-500,500]^{D}$} & $M S$ & 0 \\
\hline$f_{13}$ & Ackley & 30 and 60 & {$[-32,32]^{D}$} & $M N$ & 0 \\
\hline$f_{14}$ & Griewank & 30 and 60 & {$[-600,600]^{D}$} & $M N$ & 0 \\
\hline$f_{15}$ & Alpine & 30 and 60 & {$[-10,10]^{D}$} & $M S$ & 0 \\
\hline$f_{16}$ & Weierstrass & 30 and 60 & {$[-0.5,0.5]^{D}$} & $M S$ & 0 \\
\hline$f_{17}$ & Penalized & 30 and 60 & {$[-50,50]^{D}$} & $M N$ & 0 \\
\hline$f_{18}$ & Penalized2 & 30 and 60 & {$[-50,50]^{D}$} & $M N$ & 0 \\
\hline$f_{19}$ & Foxholes & 2 & {$[-65.536,65.536]^{D}$} & $M S$ & 1 \\
\hline$f_{20}$ & Kowalik & 4 & {$[-5,5]^{D}$} & $M N$ & $3.07 \mathrm{e}-04$ \\
\hline$f_{21}$ & $\begin{array}{l}\text { Six Hump } \\
\text { Camel Back }\end{array}$ & 2 & {$[-5,5]^{D}$} & $M N$ & -1.0316 \\
\hline$f_{22}$ & Branin & 2 & $\begin{array}{c}{[-5,10]} \\
\mathrm{x}[0,15] \\
\end{array}$ & $M S$ & 0.398 \\
\hline$f_{23}$ & Hartman3 & 3 & {$[0,1]^{D}$} & $M N$ & -3.86 \\
\hline$f_{24}$ & Hartman6 & 6 & {$[0,1]^{D}$} & $M N$ & -3.32 \\
\hline$f_{25}$ & Shekel5 & 4 & {$[0,10]^{D}$} & $M N$ & -10.15 \\
\hline$f_{26}$ & Shekel7 & 4 & {$[0,10]^{D}$} & $M N$ & -10.40 \\
\hline$f_{27}$ & Shekel10 & 4 & {$[0,10]^{D}$} & $M N$ & -10.55 \\
\hline
\end{tabular}




\begin{tabular}{|c|c|c|c|c|c|}
\hline$f_{28}$ & Fletcher Powell & 10 & {$[-\pi, \pi]^{D}$} & $M N$ & 0 \\
\hline$f_{29}$ & Michalewicz & 10 & {$[0, \pi]^{D}$} & $M S$ & -9.66015 \\
\hline$f_{30}$ & Langerman & 10 & {$[0,10]^{D}$} & $M N$ & -1.4 \\
\hline
\end{tabular}

Table 2. Comparison of $\mathrm{ABC}-\mathrm{AX}^{2}$ with basic $\mathrm{ABC}$ [2] and $\mathrm{ABC}-\mathrm{SAM}$ [15] on the standard benchmark suite functions. Best results are marked with boldface font; if not other algorithms produce similar results.

\begin{tabular}{|c|c|c|c|c|c|c|c|c|c|}
\hline \multirow{2}{*}{ No } & \multirow{2}{*}{$f_{\min }$} & \multirow{2}{*}{$D$} & \multirow{2}{*}{$G$} & \multicolumn{2}{|c|}{$\mathrm{ABC}$} & \multicolumn{2}{|c|}{ ABC-SAM } & \multicolumn{2}{|c|}{$\mathrm{ABC}-\mathbf{A} \mathbf{X}^{2}$} \\
\hline & & & & Mean & Std. Dev. & Mean & Std. Dev. & Mean & Std. Dev. \\
\hline \multirow{2}{*}{$f_{1}$} & \multirow{2}{*}{0} & 30 & 1000 & $2.45 \mathrm{e}-11$ & $7.72 \mathrm{e}-12$ & $4.18 \mathrm{e}-14$ & $5.37 \mathrm{e}-15$ & $5.51 \mathrm{e}-24$ & $3.73 \mathrm{e}-25$ \\
\hline & & 60 & 2000 & $3.75 \mathrm{e}-10$ & $2.01 \mathrm{e}-11$ & $6.09 \mathrm{e}-13$ & $7.24 \mathrm{e}-13$ & $9.43 \mathrm{e}-28$ & $7.26 \mathrm{e}-29$ \\
\hline \multirow{2}{*}{$f_{2}$} & \multirow{2}{*}{0} & 30 & 1000 & $5.05 \mathrm{e}-07$ & $1.74 \mathrm{e}-07$ & $2.47 \mathrm{e}-08$ & $2.35 \mathrm{e}-09$ & $4.23 \mathrm{e}-15$ & $3.54 \mathrm{e}-16$ \\
\hline & & 60 & 2000 & $5.58 \mathrm{e}-06$ & $1.17 \mathrm{e}-06$ & $5.06 \mathrm{e}-07$ & $2.97 \mathrm{e}-07$ & $2.98 \mathrm{e}-17$ & $1.07 e-17$ \\
\hline \multirow{2}{*}{$f_{3}$} & \multirow{2}{*}{0} & 30 & 1000 & $4.18 \mathrm{e}+01$ & 5.90 & $1.69 \mathrm{e}+01$ & 1.43 & $6.60 \mathrm{e}-02$ & $5.21 \mathrm{e}-03$ \\
\hline & & 60 & 2000 & $7.31 \mathrm{e}+01$ & 6.88 & $3.10 \mathrm{e}+01$ & 5.12 & 2.78 & $\mathbf{0 . 7 7}$ \\
\hline \multirow{2}{*}{$f_{4}$} & \multirow{2}{*}{0} & 30 & 1000 & $8.32 \mathrm{e}-10$ & $9.75 \mathrm{e}-11$ & $3.95 \mathrm{e}-12$ & $5.77 \mathrm{e}-13$ & $3.42 \mathrm{e}-16$ & $8.83 e-18$ \\
\hline & & 60 & 2000 & $4.50 \mathrm{e}-09$ & $5.64 \mathrm{e}-10$ & $7.54 \mathrm{e}-11$ & $2.14 \mathrm{e}-11$ & $8.84 \mathrm{e}-20$ & $5.45 e-21$ \\
\hline$f_{5}$ & 0 & 24 & 1000 & $6.61 \mathrm{e}+00$ & $1.07 \mathrm{e}+00$ & $9.24 \mathrm{e}-01$ & $2.08 \mathrm{e}-01$ & $2.23 \mathrm{e}-02$ & $3.75 \mathrm{e}-03$ \\
\hline \multirow{2}{*}{$f_{6}$} & \multirow{2}{*}{0} & 30 & 1000 & $6.67 \mathrm{e}-01$ & $1.21 \mathrm{e}-08$ & $2.16 \mathrm{e}-03$ & $6.37 \mathrm{e}-04$ & $5.91 \mathrm{e}-05$ & $5.67 \mathrm{e}-06$ \\
\hline & & 60 & 2000 & $6.66 \mathrm{e}-01$ & $1.05 \mathrm{e}-07$ & $7.76 \mathrm{e}-02$ & $1.63 \mathrm{e}-02$ & $8.33 \mathrm{e}-05$ & $1.71 \mathrm{e}-05$ \\
\hline \multirow{2}{*}{$f_{7}$} & \multirow{2}{*}{0} & 30 & 1000 & $4.25 \mathrm{e}-01$ & $1.18 \mathrm{e}-01$ & $2.28 \mathrm{e}+01$ & 3.75 & $2.39 \mathrm{e}+01$ & 3.66 \\
\hline & & 60 & 2000 & $2.02 \mathrm{e}-01$ & $6.92 \mathrm{e}-02$ & $4.96 \mathrm{e}+01$ & 7.80 & $5.15 \mathrm{e}+01$ & 7.69 \\
\hline \multirow{2}{*}{$f_{8}$} & 0 & 30 & 1000 & 0 & 0 & 0 & 0 & 0 & 0 \\
\hline & 0 & 60 & 2000 & 0 & 0 & 0 & 0 & 0 & 0 \\
\hline$f$ & 0 & 30 & 1000 & $8.60 \mathrm{e}-13$ & $8.32 \mathrm{e}-13$ & $3.66 \mathrm{e}-16$ & $1.44 \mathrm{e}-17$ & $8.87 e-34$ & $6.78 \mathrm{e}-35$ \\
\hline$J_{9}$ & 0 & 60 & 2000 & $9.31 \mathrm{e}-12$ & $7.17 \mathrm{e}-12$ & $4.76 \mathrm{e}-15$ & $5.32 \mathrm{e}-16$ & 6.31e-32 & $2.16 \mathrm{e}-33$ \\
\hline & 0 & 30 & 1000 & $1.72 \mathrm{e}-14$ & $1.56 \mathrm{e}-14$ & $1.26 \mathrm{e}-16$ & $2.11 \mathrm{e}-17$ & $4.68 \mathrm{e}-24$ & $9.03 \mathrm{e}-26$ \\
\hline$J_{10}$ & 0 & 60 & 2000 & $2.84 \mathrm{e}-13$ & $8.01 \mathrm{e}-14$ & $8.55 \mathrm{e}-15$ & $3.15 \mathrm{e}-16$ & $6.12 \mathrm{e}-31$ & $8.67 e-33$ \\
\hline & & 30 & 1000 & $2.33 \mathrm{e}-08$ & $7.49 \mathrm{e}-09$ & $4.60 \mathrm{e}-10$ & $8.85 \mathrm{e}-11$ & $1.04 \mathrm{e}-13$ & $3.16 \mathrm{e}-14$ \\
\hline$f_{11}$ & 0 & 60 & 2000 & $6.64 \mathrm{e}-07$ & $1.51 \mathrm{e}-07$ & $6.80 \mathrm{e}-09$ & $8.77 \mathrm{e}-10$ & $4.25 \mathrm{e}-13$ & $7.32 \mathrm{e}-14$ \\
\hline & -12569.5 & 30 & 1000 & -11346.79 & $2.77 \mathrm{e}+02$ & -12416.19 & $4.02 \mathrm{e}+01$ & -12569.48 & $1.50 \mathrm{e}-02$ \\
\hline$f_{12}$ & -25138.9 & 60 & 2000 & -22530.82 & $4.08 \mathrm{e}+02$ & -23805.93 & $2.84 \mathrm{e}+02$ & -25016.6 & $1.89 \mathrm{e}+01$ \\
\hline & & 30 & 1000 & $2.93 \mathrm{e}-06$ & $3.38 \mathrm{e}-07$ & $9.26 \mathrm{e}-08$ & $1.89 \mathrm{e}-08$ & $8.13 \mathrm{e}-13$ & $6.71 \mathrm{e}-14$ \\
\hline$f_{13}$ & 0 & 60 & 2000 & $4.65 \mathrm{e}-06$ & $1.07 \mathrm{e}-06$ & $2.07 \mathrm{e}-08$ & $3.55 \mathrm{e}-08$ & $3.62 \mathrm{e}-14$ & $1.15 e-15$ \\
\hline & & 30 & 1000 & $4.55 \mathrm{e}-08$ & $6.54 \mathrm{e}-09$ & $8.36 \mathrm{e}-10$ & $5.08 \mathrm{e}-11$ & $5.63 \mathrm{e}-23$ & $7.35 \mathrm{e}-25$ \\
\hline$f_{14}$ & 0 & 60 & 2000 & $8.01 \mathrm{e}-07$ & $2.64 \mathrm{e}-07$ & $1.56 \mathrm{e}-10$ & $6.90 \mathrm{e}-11$ & $7.04 \mathrm{e}-31$ & $5.77 \mathrm{e}-32$ \\
\hline & 0 & 30 & 1000 & $3.34 \mathrm{e}-04$ & $3.76 \mathrm{e}-05$ & $2.22 \mathrm{e}-08$ & $3.93 \mathrm{e}-09$ & $8.56 \mathrm{e}-13$ & $1.56 \mathrm{e}-13$ \\
\hline$f_{15}$ & 0 & 60 & 2000 & $7.49 \mathrm{e}-03$ & $9.58 \mathrm{e}-04$ & $1.17 \mathrm{e}-08$ & $2.35 \mathrm{e}-09$ & $5.37 \mathrm{e}-13$ & $1.25 \mathrm{e}-13$ \\
\hline & & 30 & 1000 & $3.36 \mathrm{e}-01$ & $9.58 \mathrm{e}-02$ & $5.78 \mathrm{e}-04$ & $6.31 \mathrm{e}-05$ & $6.46 \mathrm{e}-09$ & $8.32 \mathrm{e}-10$ \\
\hline$f_{16}$ & 0 & 60 & 2000 & $8.99 \mathrm{e}-01$ & $3.09 \mathrm{e}-01$ & $9.20 \mathrm{e}-03$ & $4.03 \mathrm{e}-03$ & $5.38 \mathrm{e}-08$ & $9.19 \mathrm{e}-10$ \\
\hline & & 30 & 1000 & $5.47 \mathrm{e}-12$ & $2.09 \mathrm{e}-13$ & $2.78 \mathrm{e}-12$ & $8.89 \mathrm{e}-13$ & $3.85 \mathrm{e}-14$ & $4.93 \mathrm{e}-15$ \\
\hline$f_{17}$ & 0 & 60 & 2000 & $7.47 \mathrm{e}-12$ & $1.74 \mathrm{e}-12$ & $1.32 \mathrm{e}-12$ & $5.15 \mathrm{e}-13$ & $3.50 \mathrm{e}-14$ & $2.60 \mathrm{e}-15$ \\
\hline & O & 30 & 1000 & $2.63 \mathrm{e}-03$ & $1.89 \mathrm{e}-04$ & $3.06 \mathrm{e}-02$ & $8.59 \mathrm{e}-03$ & $2.33 \mathrm{e}-21$ & $7.55 \mathrm{e}-22$ \\
\hline$f_{18}$ & 0 & 60 & 2000 & $2.66 \mathrm{e}-03$ & $7.90 \mathrm{e}-04$ & $5.11 \mathrm{e}-02$ & $7.39 \mathrm{e}-03$ & $7.52 \mathrm{e}-26$ & $1.29 \mathrm{e}-26$ \\
\hline$f_{19}$ & 1 & 2 & 100 & 1.04 & 0.04 & 1.03 & 0.03 & 1.01 & 0.01 \\
\hline$f_{20}$ & $3.07 \mathrm{e}-04$ & 4 & 100 & $5.98 \mathrm{e}-04$ & $7.22 \mathrm{e}-05$ & $4.32 \mathrm{e}-04$ & $1.09 \mathrm{e}-05$ & $3.10 \mathrm{e}-04$ & $8.73 e-06$ \\
\hline$f_{21}$ & -1.0316 & 2 & 100 & -1.0316 & 0 & -1.0316 & 0 & -1.0316 & 0 \\
\hline$f_{22}$ & 0.398 & 2 & 100 & 0.398 & $7.12 \mathrm{e}-08$ & 0.398 & $2.75 \mathrm{e}-07$ & 0.398 & $1.83 \mathrm{e}-07$ \\
\hline$f_{23}$ & -3.86 & 3 & 100 & -3.86 & $7.09 \mathrm{e}-07$ & -3.86 & $1.54 \mathrm{e}-08$ & -3.86 & $6.77 \mathrm{e}-10$ \\
\hline$f_{24}$ & -3.32 & 6 & 100 & -3.32 & $4.74 \mathrm{e}-13$ & -3.32 & $6.26 \mathrm{e}-14$ & -3.32 & $2.61 \mathrm{e}-15$ \\
\hline$f_{25}$ & -10.15 & 4 & 100 & -9.61 & 0.14 & -10.14 & $3.68 \mathrm{e}-07$ & -10.15 & $9.15 e-08$ \\
\hline$f_{26}$ & -10.40 & 4 & 100 & -10.40 & $8.61 \mathrm{e}-03$ & -10.40 & $7.94 \mathrm{e}-03$ & -10.40 & $2.56 \mathrm{e}-03$ \\
\hline$f_{27}$ & -10.54 & 4 & 100 & -10.52 & 0.08 & -10.54 & $6.77 \mathrm{e}-07$ & $-\mathbf{1 0 . 5 5}$ & $7.84 \mathrm{e}-08$ \\
\hline$f_{28}$ & 0 & 10 & 100 & 13.77 & 3.80 & 4.02 & 0.39 & $4.19 \mathrm{e}-01$ & $6.54 \mathrm{e}-02$ \\
\hline$f_{29}$ & -9.66015 & 10 & 100 & -9.66015 & 0 & -9.66015 & 0 & -9.66015 & 0 \\
\hline
\end{tabular}




\begin{tabular}{|c|c|c|c|c|c|c|c|c|c|}
\hline$f_{30}$ & -1.4 & 10 & 100 & -0.78 & 0.09 & -1.04 & 0.06 & -1.28 & $\mathbf{0 . 0 3}$ \\
\hline \multirow{3}{*}{\multicolumn{2}{|c|}{$\underset{(t \text {-Test })}{\text { Summary }}$}} & \multicolumn{2}{|c|}{+} & \multicolumn{2}{|c|}{20} & \multicolumn{2}{|c|}{19} & & \\
\hline & & & & \multicolumn{2}{|c|}{1} & \multicolumn{2}{|c|}{0} & & \\
\hline & & & & \multicolumn{2}{|c|}{9} & \multicolumn{2}{|c|}{11} & & \\
\hline
\end{tabular}
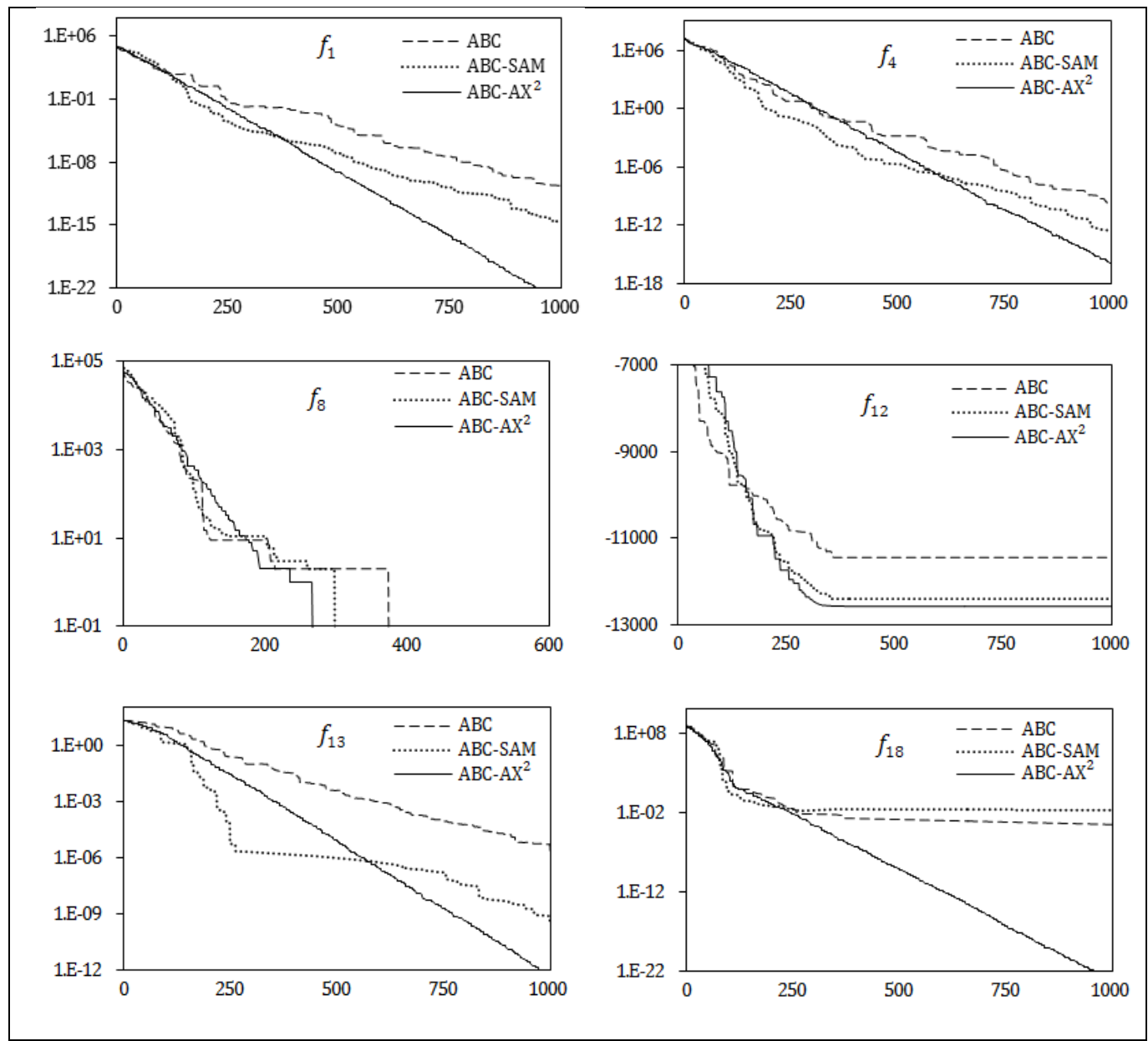

Fig. 4. Convergence characteristics of $\mathrm{ABC}, \mathrm{ABC}-\mathrm{SAM}$ and $\mathrm{ABC}-\mathrm{AX} \mathrm{X}^{2}$ on three unimodal $\left(f_{1}, f_{4}, f_{8}\right)$ and three multimodal $\left(f_{12}, f_{13}, f_{18}\right)$ functions. The vertical axis is the function value and the horizontal axis is the number of cycles elapsed.

- ABC-SAM vs. ABC-AX ${ }^{2}$ : On all of the 30 functions, $\mathrm{ABC}-\mathrm{AX}^{2}$ performs either better than or as well as ABC-SAM. For the high dimensional functions $f_{1}-f_{18}$, $\mathrm{ABC}-\mathrm{AX}^{2}$ significantly outperforms $\mathrm{ABC}-\mathrm{SAM}$ on as many as 16 functions and shows similar performance on two $\left(f_{7}\right.$ and $\left.f_{8}\right)$. On most (nine out of twelve) of the low dimensional functions $f_{19}-f_{30}$, both perform equally well, but $\mathrm{ABC}-\mathrm{AX}^{2}$ performs better on the remaining three.

- For almost all the functions, $\mathrm{ABC}-\mathrm{AX}^{2}$ shows very low standard deviation of its results. This indicates its high degree of consistency and robustness for all these benchmark functions.

- The '+', '-' and ' $\approx$ ' symbols at the bottom rows count the number of functions where $\mathrm{ABC}-\mathrm{AX}^{2}$ produces significantly better, worse and similar results, respectively compared to $\mathrm{ABC}$ or $\mathrm{ABC}-\mathrm{SAM}$. Out of 30 functions, $\mathrm{ABC}-\mathrm{AX} \mathrm{X}^{2}$ performs significantly better than $\mathrm{ABC}$ and $\mathrm{ABC}-\mathrm{SAM}$ on 20 and 19 functions, shows similar performance on 9 and 11 functions, while $\mathrm{ABC}$ performs better on one function $\left(f_{7}\right)$ only. So the overall performance of $\mathrm{ABC}-\mathrm{AX}^{2}$ is much better than others.

Fig. 4 shows the convergence graphs of $\mathrm{ABC}, \mathrm{ABC}-\mathrm{SAM}$ and $\mathrm{ABC}-\mathrm{AX}^{2}$ for three unimodal $\left(f_{1}, f_{4}, f_{8}\right)$ and three multimodal $\left(f_{12}, f_{13}, f_{18}\right)$ functions with $D=30$. ABC-AX ${ }^{2}$ shows far better convergence characteristics than its counterparts for all these functions. For example, consider the functions $f_{12}$ and $f_{18}$, where both $\mathrm{ABC}$ and $\mathrm{ABC}-\mathrm{SAM}$ converges to a local minimum and gets stuck there till the end of their execution. In contrast, $\mathrm{ABC}-\mathrm{AX}^{2}$ easily reaches the global minimum for $f_{12}$ and shows no sign of fitness stagnation for $f_{18}$, even after reaching the vicinity of the global minimum. For some functions, e.g., $f_{1}, f_{4}, f_{13}$ and $f_{18}$, ABC-SAM initially shows somewhat higher convergence speed than $\mathrm{ABC}-\mathrm{AX}^{2}$, but eventually it either gets stuck at local optima $\left(f_{13}, f_{18}\right)$ or gradually slows down $\left(f_{1}, f_{4}\right)$ and at the end, $\mathrm{ABC}-\mathrm{AX}{ }^{2}$ shows significantly higher convergence speed than both $\mathrm{ABC}$ and ABC-SAM. Fig. 4 shows that $\mathrm{ABC}-\mathrm{AX}^{2}$ has always reached 
very close proximity to the global minimum, while $\mathrm{ABC}$ and ABC-SAM can get stuck at several intermediate local optima (note the semi-flat and flat regions of the plot of $\mathrm{ABC}$ in $f_{4}$ and $\mathrm{ABC}-\mathrm{SAM}$ in $f_{13}$ ). Previously Table 2 considered the performance of all three algorithms to be similar on $f_{8}$, but Fig. 4 now reveals that $\mathrm{ABC}-\mathrm{AX}^{2}$ actually reaches the global minimum of $f_{8}$ much earlier than both $\mathrm{ABC}-\mathrm{SAM}$ and $\mathrm{ABC}$.

Table 3. Comparison of $\mathrm{ABC}-\mathrm{AX}^{2}$ with the $\mathrm{CABC}$ [16] variants. The boldface font marks the best performance for each function. The,+- and $\approx$ counts the number of instances where $\mathrm{ABC}-\mathrm{AX} \mathrm{X}^{2}$ performs better, worse and similar, respectively.

\begin{tabular}{|c|c|c|c|c|c|c|}
\hline \multirow{2}{*}{ Function } & \multicolumn{2}{|c|}{ CABC_S } & \multicolumn{2}{|c|}{ CABC_H } & \multicolumn{2}{|c|}{$A B C-A X^{2}$} \\
\hline & Mean & Std. Dev. & Mean & Std. Dev. & Mean & Std. Dev. \\
\hline$f_{1}$ & $3.30 \mathrm{e}-19$ & $2.00 \mathrm{e}-19$ & $5.92 \mathrm{e}-18$ & $3.56 \mathrm{e}-18$ & $7.22 e-51$ & $4.08 \mathrm{e}-52$ \\
\hline$f_{7}$ & $6.33 e+00$ & $7.68 \mathrm{e}+00$ & $4.80 \mathrm{e}-01$ & $8.55 \mathrm{e}-01$ & $5.94 \mathrm{e}+00$ & $4.35 \mathrm{e}+00$ \\
\hline$f_{10}$ & 0 & 0 & $\mathbf{0}$ & 0 & $8.56 \mathrm{e}-54$ & $3.83 \mathrm{e}-55$ \\
\hline$f_{12}$ & $1.30 \mathrm{e}-04$ & $5.21 \mathrm{e}-06$ & $1.27 \mathrm{e}-04$ & 0 & $1.04 \mathrm{e}-06$ & $7.98 \mathrm{e}-08$ \\
\hline$f_{13}$ & $1.83 \mathrm{e}-14$ & $9.86 \mathrm{e}-15$ & $8.35 \mathrm{e}-15$ & $4.13 e-15$ & $7.14 \mathrm{e}-18$ & $7.83 e-19$ \\
\hline$f_{14}$ & $4.42 \mathrm{e}-02$ & $2.99 \mathrm{e}-02$ & $7.96 \mathrm{e}-03$ & $9.06 \mathrm{e}-03$ & $3.84 e-54$ & $2.04 \mathrm{e}-55$ \\
\hline+ & \multicolumn{2}{|c|}{4} & \multicolumn{2}{|c|}{4} & & \\
\hline- & \multicolumn{2}{|c|}{1} & \multicolumn{2}{|c|}{2} & & \\
\hline$\approx$ & \multicolumn{2}{|c|}{1} & \multicolumn{2}{|c|}{0} & & \\
\hline
\end{tabular}

Table 4. Comparison between $\mathrm{ABC}-\mathrm{AX}^{2}$ and $\mathrm{DABC}$ [17]. Best results are marked with bold font; if not both the algorithms produce identical results.

\begin{tabular}{|c|c|c|c|c|c|}
\hline \multirow{2}{*}{ Function } & \multirow{2}{*}{$D$} & \multicolumn{2}{|c|}{ DABC } & \multicolumn{2}{|c|}{$\mathbf{A B C}-\mathbf{A} \mathbf{X}^{2}$} \\
\hline & & Mean & Std. Dev. & Mean & Std. Dev. \\
\hline \multirow{2}{*}{$f_{1}$} & 10 & $2.01 \mathrm{e}-17$ & $5.63 \mathrm{e}-17$ & $\mathbf{0}$ & 0 \\
\hline & 30 & $2.01 \mathrm{e}-16$ & $2.85 \mathrm{e}-17$ & $7.26 \mathrm{e}-51$ & $6.47 \mathrm{e}-52$ \\
\hline \multirow{2}{*}{$f_{7}$} & 10 & $2.73 e-03$ & $7.04 \mathrm{e}-03$ & $9.46 e-02$ & $8.22 \mathrm{e}-03$ \\
\hline & 30 & $1.42 \mathrm{e}-02$ & $2.53 \mathrm{e}-02$ & $5.88 \mathrm{e}-01$ & $8.32 \mathrm{e}-02$ \\
\hline \multirow{2}{*}{$f_{10}$} & 10 & 0 & 0 & 0 & 0 \\
\hline & 30 & 0 & 0 & 0 & 0 \\
\hline \multirow{2}{*}{$f_{14}$} & 10 & 0 & 0 & 0 & 0 \\
\hline & 30 & $2.59 \mathrm{e}-16$ & $1.22 \mathrm{e}-16$ & $1.78 \mathrm{e}-50$ & $5.28 \mathrm{e}-51$ \\
\hline \multicolumn{2}{|c|}{+} & \multicolumn{2}{|c|}{2} & & \\
\hline \multicolumn{2}{|c|}{-} & \multicolumn{2}{|c|}{1} & & \\
\hline \multicolumn{2}{|c|}{$\approx$} & \multicolumn{2}{|c|}{1} & & \\
\hline
\end{tabular}

Table 5. Comparison between $\mathrm{ABC}-\mathrm{AX}^{2}$ and $\mathrm{ChABC}$ [18]. Best results are marked with bold font.

\begin{tabular}{|c|c|c|c|c|c|}
\hline \multirow{2}{*}{ Function } & \multirow{2}{*}{$\boldsymbol{D}$} & \multicolumn{2}{|c|}{ CHABC } & \multicolumn{2}{c|}{ ABC-AX } \\
\cline { 3 - 6 } & & Mean & Std. Dev. & Mean & Std. Dev. \\
\hline$f_{1}$ & 30 & $2.99 \mathrm{e}-16$ & $3.54 \mathrm{e}-17$ & $\mathbf{9 . 7 6 e}-119$ & $\mathbf{7 . 1 7 e}-120$ \\
\hline$f_{7}$ & 30 & $6.33 \mathrm{e}-02$ & $8.96 \mathrm{e}-02$ & $\mathbf{8 . 4 8 e}-\mathbf{0 7}$ & $\mathbf{4 . 9 9 e - 0 8}$ \\
\hline$f_{10}$ & 30 & $\mathbf{0}$ & $\mathbf{0}$ & $8.60 \mathrm{e}-129$ & $5.14 \mathrm{e}-130$ \\
\hline$f_{12}$ & 30 & $3.81 \mathrm{e}-04$ & $2.07 \mathrm{e}-04$ & $\mathbf{5 . 3 2 e}-\mathbf{0 6}$ & $\mathbf{6 . 0 4 e}-\mathbf{0 7}$ \\
\hline$f_{13}$ & 30 & $2.93 \mathrm{e}-14$ & $2.99 \mathrm{e}-15$ & $\mathbf{2 . 4 8 e}-17$ & $\mathbf{4 . 0 9 e}-18$ \\
\hline$f_{14}$ & 30 & $2.70 \mathrm{e}-16$ & $6.20 \mathrm{e}-17$ & $\mathbf{1 . 8 5 e}-129$ & $\mathbf{5 . 7 5 e}-130$ \\
\hline \hline \multicolumn{7}{|c|}{5} \\
\hline
\end{tabular}




\begin{tabular}{|l|l|}
\hline- & 1 \\
\cline { 1 - 2 } & 0 \\
\hline
\end{tabular}

Table 6. Comparison between $\mathrm{ABC}-\mathrm{AX}^{2}$ and GABC [20]. Best results are marked with bold font.

\begin{tabular}{|c|c|c|c|c|c|c|c|}
\hline \multirow{2}{*}{ Function } & \multirow{2}{*}{$D$} & \multicolumn{2}{|c|}{$\operatorname{GABC}(C=1.0)$} & \multicolumn{2}{|c|}{$\operatorname{GABC}(C=1.5)$} & \multicolumn{2}{|c|}{$A B C-A X^{2}$} \\
\hline & & Mean & Std. Dev. & Mean & Std. Dev. & Mean & Std. Dev. \\
\hline \multirow{2}{*}{$f_{1}$} & 30 & $4.31 \mathrm{e}-16$ & $7.49 \mathrm{e}-17$ & $4.17 \mathrm{e}-16$ & $7.36 \mathrm{e}-17$ & $9.66 \mathrm{e}-105$ & $8.05 e-106$ \\
\hline & 60 & $1.43 \mathrm{e}-15$ & $1.43 \mathrm{e}-16$ & $1.43 \mathrm{e}-15$ & $1.37 \mathrm{e}-16$ & $1.98 e-36$ & $3.58 e-37$ \\
\hline \multirow{2}{*}{$f_{7}$} & 2 & $3.93 \mathrm{e}-04$ & $4.45 \mathrm{e}-04$ & $1.68 \mathrm{e}-04$ & $1.45 \mathrm{e}-04$ & $7.88 \mathrm{e}-03$ & $5.35 \mathrm{e}-04$ \\
\hline & 3 & $2.63 \mathrm{e}-03$ & $2.11 e-03$ & $2.65 \mathrm{e}-03$ & $2.22 \mathrm{e}-03$ & $2.13 \mathrm{e}-01$ & $6.12 \mathrm{e}-02$ \\
\hline \multirow{2}{*}{$f_{10}$} & 30 & $9.47 \mathrm{e}-15$ & $2.15 \mathrm{e}-14$ & $1.32 \mathrm{e}-14$ & $2.44 \mathrm{e}-14$ & $6.30 \mathrm{e}-108$ & $1.52 \mathrm{e}-109$ \\
\hline & 60 & $4.16 \mathrm{e}-13$ & $1.77 \mathrm{e}-13$ & $3.52 \mathrm{e}-13$ & $1.24 \mathrm{e}-13$ & $7.42 \mathrm{e}-40$ & $3.75 e-41$ \\
\hline \multirow{2}{*}{$f_{13}$} & 30 & $3.31 \mathrm{e}-14$ & $2.90 \mathrm{e}-15$ & $3.21 \mathrm{e}-14$ & $3.25 \mathrm{e}-15$ & $4.04 \mathrm{e}-19$ & $8.28 \mathrm{e}-20$ \\
\hline & 60 & $1.04 \mathrm{e}-13$ & $1.07 \mathrm{e}-14$ & $1.00 \mathrm{e}-13$ & $6.08 \mathrm{e}-15$ & $1.63 e-17$ & $2.66 \mathrm{e}-18$ \\
\hline \multirow{2}{*}{$f_{14}$} & 30 & $8.88 \mathrm{e}-17$ & $8.45 \mathrm{e}-17$ & $2.96 \mathrm{e}-17$ & $4.99 \mathrm{e}-17$ & $9.50 \mathrm{e}-101$ & $2.00 \mathrm{e}-102$ \\
\hline & 60 & $9.47 e-16$ & $7.84 \mathrm{e}-16$ & $7.54 \mathrm{e}-17$ & $4.12 \mathrm{e}-16$ & $1.81 \mathrm{e}-33$ & $3.25 \mathrm{e}-34$ \\
\hline \multicolumn{2}{|l|}{+} & \multicolumn{2}{|c|}{4} & \multicolumn{2}{|c|}{4} & & \\
\hline \multicolumn{2}{|c|}{-} & \multicolumn{2}{|c|}{1} & \multicolumn{2}{|c|}{1} & & \\
\hline \multicolumn{2}{|c|}{$\approx$} & \multicolumn{2}{|c|}{0} & \multicolumn{2}{|c|}{0} & & \\
\hline
\end{tabular}

Table 7. Comparison of $\mathrm{ABC}-\mathrm{AX}^{2}$ with $\mathrm{HJABC}$ [21] based on convergence speed. Best results are marked with bold font.

\begin{tabular}{|c|c|c|c|}
\hline \multirow{2}{*}{ Function } & \multirow{2}{*}{$D$} & \multicolumn{2}{|c|}{ Number of function evaluations } \\
\hline & & НJАВС & $A B C-A X^{2}$ \\
\hline$f_{1}$ & 30 & 18322 & 13805 \\
\hline$f_{2}$ & 30 & 12509 & 17987 \\
\hline$f_{3}$ & 30 & 120315 & - \\
\hline$f_{4}$ & 30 & 43939 & 35502 \\
\hline$f_{7}$ & 30 & 102718 & - \\
\hline$f_{8}$ & 30 & 17755 & 13986 \\
\hline$f_{9}$ & 30 & - & 12230 \\
\hline$f_{10}$ & 30 & 15376 & 20713 \\
\hline$f_{13}$ & 30 & 54497 & 42609 \\
\hline$f_{14}$ & 30 & 56855 & 31582 \\
\hline$f_{15}$ & 30 & 99686 & 81678 \\
\hline \multicolumn{2}{|c|}{+} & 7 & \\
\hline \multicolumn{2}{|c|}{-} & 4 & \\
\hline \multicolumn{2}{|c|}{$\approx$} & 0 & \\
\hline
\end{tabular}

\subsection{Comparison with other ABC-variants}

In this section $\mathrm{ABC}-\mathrm{AX}^{2}$ is compared with some other recent variants of $A B C$, such as the cooperative $A B C$ (CABC) [16], $\mathrm{ABC}$ with diversity strategy [17], chaotic $\mathrm{ABC}$ (CHABC) [18], gbest-guided ABC (GABC) [20] and Hooke Jeeves ABC
(HJABC) [21]. The first three variants (e.g., [16]-[18]) increase the degree of explorations, while the last two variants (e.g., [20]-[21]) increase the intensity of exploitations.

First, $\mathrm{ABC}-\mathrm{AX}^{2}$ is compared with $\mathrm{CABC}$ [16], which is a cooperative variant of the basic $\mathrm{ABC}$ algorithm. $\mathrm{CABC}$ has 
been introduced in two different versions - CABC_S and CABC_H. In order to perform more explorations, CABC_S decomposes the search space into multiple sub-spaces and employs different bee colonies to search and explore the different sub-spaces. The other variant, $\mathrm{CABC} \_\mathrm{H}$ tries to perform more exploitations than $\mathrm{CABC}_{-} \mathrm{S}$ by repeatedly alternating between explorative CABC_S and exploitative $\mathrm{ABC}$. For comparison, $\mathrm{ABC}-\mathrm{AX}^{2}$ is re-implemented with the same settings [16] - SN=40, no. of function evaluations $F E=100,000$ and limit $=S N * D$. Table 3 shows that $\mathrm{ABC}-\mathrm{AX}^{2}$ significantly outperforms both the $\mathrm{CABC}$ variants on four out of the six benchmark functions, while CABC_S and CABC_H perform better on one or two functions only. So the overall performance of $\mathrm{ABC}-\mathrm{AX}^{2}$ is better than the $\mathrm{CABC}$ variants.

The next comparison is made between $\mathrm{ABC}-\mathrm{AX}^{2}$ and $\mathrm{DABC}$ [17]. DABC tries to maintain sufficient amount of diversity among the candidate solutions to allow more search space explorations. DABC regularly measures the existing population diversity $d$ and employs either its explorative or exploitative perturbation based on the value of $d$. ABC-AX ${ }^{2}$ is re-implemented with $S N=20, M C N=5000$ and limit $=100$ to compare with DABC. Results presented in Table 4 show that $\mathrm{ABC}-\mathrm{AX}^{2}$ performs better than $\mathrm{DABC}$ on two out of four functions $\left(f_{1}\right.$ and $\left.f_{14}\right)$, shows similar performance on one $\left(f_{10}\right)$, while DABC performs better on the remaining one function $\left(f_{7}\right)$ only. The reason may be that DABC completely relies on its estimated value of population diversity $d$ to choose between explorations and exploitations, while there is no accurate metric for diversity. Besides, DABC uses a naïve strategy of fixed threshold diversity value ( $d_{\text {low }}$ in [17]), which may cause repeated oscillations between conflicting explorations and exploitations to reduce convergence speed.

Next, $\mathrm{ABC}-\mathrm{AX}^{2}$ is compared with the Chaotic $\mathrm{ABC}$ (CHABC) [18] algorithm. CHABC employs chaotic search behavior during perturbations to produce new food positions from the existing ones. Chaotic dynamics are produced by the logistic equations (eq. (4)-(7) in [18]) which provide a simple mechanism to escape from local minima and avoid premature convergence. For comparison, $\mathrm{ABC}-\mathrm{AX}^{2}$ is executed for 5000 cycles with population size of 70 and limit $=200$, as suggested in [18]. Results (Table 5) show that $\mathrm{ABC}-\mathrm{AX}^{2}$ outperforms $\mathrm{CHABC}$ on as many as five out of the six functions, while CHABC performs better on the remaining one function $\left(f_{10}\right)$ only. The reason may be that $\mathrm{CHABC}$ employs same chaotic strategy uniformly for all the candidate solutions across the population, without considering their individual exploitative/explorative requirement, while $\mathrm{ABC}-\mathrm{AX}^{2}$ considers and customizes the degree of explorations and exploitations separately for every candidate solution.

Next, $\mathrm{ABC}-\mathrm{AX}^{2}$ is compared with GABC [20], which is an exploitative $\mathrm{ABC}$-variant that tries to improve the convergence speed by using the information of the global best solution found so far into the perturbation scheme (1). $\mathrm{ABC}-\mathrm{AX}^{2}$ is executed with the same settings [20] and results are presented in Table 6 . In [20], GABC is tested with several values of its parameter $C$, but the best results are always observed with $C=1.0$ or 1.5 , so Table 6 includes both the results. Results show that $\mathrm{ABC}-\mathrm{AX}^{2}$ outperforms $\mathrm{GABC}$ on four out of the five functions, while GABC performs better on the remaining one $\left(f_{7}\right)$ only. The reason may be that the perturbation operation of GABC becomes too exploitative by pushing its candidate solutions towards the best solution found so far. Increased exploitations, at the cost of reduced explorations, may improve the final solution quality for the unimodal and low dimensional function $f_{7}$, but is likely to fail for the other four multimodal functions in Table 6 .

Next, $\mathrm{ABC}-\mathrm{AX}^{2}$ is compared with HJABC [21], which is a hybrid $\mathrm{ABC}$-variant that intensifies the degree of exploitations by hybridizing basic $\mathrm{ABC}$ with an efficient local search technique (i.e., Hooke Jeeves pattern search). Table 7 compares $\mathrm{ABC}-\mathrm{AX}^{2}$ and $\mathrm{HJABC}$ based on the number of function evaluations (NFE) required to achieve a predefined level of accuracy. Both $\mathrm{ABC}-\mathrm{AX}^{2}$ and HJABC are run with $S N=25$ and limit $=S N * D$, until either NFE reaches a predefined maximum value $\left(N F E_{\max }\right)$ or the algorithm reaches as accuracy of $\varepsilon$ around the global minimum. As suggested in [21], $\mathrm{ABC}-\mathrm{AX}^{2}$ used $\varepsilon=10^{-8}$ with $N F E_{\max }=300000$. For seven out of the eleven functions in Table $7, \mathrm{ABC}-\mathrm{AX}^{2}$ performs better than $\mathrm{HJABC}$, by showing a faster convergence speed, while HJABC performs better on the remaining four. However, ABC-AX ${ }^{2}$ can't achieve the predefined level of accuracy within $N F E_{\max }$ function evaluations for two functions $\left(f_{3}\right.$ and $\left.f_{7}\right)$, while $\mathrm{HJABC}$ fails to do so only for one function $\left(f_{9}\right)$. In short, the overall performance of $\mathrm{ABC}-\mathrm{AX}^{2}$ is quite comparable to HJABC. The reason that HJABC often requires larger number of function evaluations, even after using the efficient Hooke Jeeves local searcher [21], may be that $\mathrm{HJABC}$ regularly tries to find an appropriate search direction by exploring along the axis directions only, exploring just one variable at a time, which is not suitable for the non-separable problems.

\section{CONCLUSION AND SUGGESTION FOR FURTHER STUDY}

This paper introduces $\mathrm{ABC}-\mathrm{AX}^{2}$ - an improvement of the basic $\mathrm{ABC}$ algorithm [2] that tries to adaptively control the degree of explorations and exploitations, separately for each candidate solution. $\mathrm{ABC}-\mathrm{AX}^{2}$ includes three control parameters $-p_{i}, q_{i}$ and $\boldsymbol{\eta}_{i}$ within each candidate solution $\boldsymbol{x}_{i}$ and employs adaptive and self-adaptive techniques to adapt their values gradually. The control parameter $p_{i}$ controls the proportion of exploitative and explorative perturbations on $\boldsymbol{x}_{i}$ and is gradually adapted by $\mathrm{ABC}-\mathrm{AX}^{2}$ based on the previous successes and failures of the exploitative and explorative perturbations on $\boldsymbol{x}_{\boldsymbol{i}}$. The other two control parameters $-q_{i}$ and $\boldsymbol{\eta}_{i}$ control the perturbation rate and perturbation scaling factors for $\boldsymbol{x}_{i}$ and they have to go through gradual self-adaptation, using (8) and (10) respectively.

$\mathrm{ABC}-\mathrm{AX}^{2}$ significantly differs from most other existing variants of $A B C$ algorithm. Most $A B C$-variants view exploitations and explorations as conflicting operations, so they try to improve either the local exploitations (e.g., GABC [20], HJABC [21]) or the global explorations (e.g., CABC [16], DABC [17], CHABC [18]) of the basic ABC algorithm, without trying to establish a proper balance between exploitations and explorations. In contrast, $\mathrm{ABC}-\mathrm{AX}^{2}$ considers exploitations and explorations to be complementary, rather than conflicting, operations and try to achieve some degree of both exploitations and explorations throughout the entire optimization procedure. For example, $\mathrm{ABC}-\mathrm{AX}^{2}$ keeps the value of $p_{i}$ always within $[0.1,0.9]$ to avoid the complete domination by either exploitative or explorative perturbations. Also, $\mathrm{ABC}-\mathrm{AX}^{2}$ uses fixed values of $u_{1}$ and $u_{2}$ (e.g., 0.1 and 0.5 , respectively, as in the current implementation), so there is always significant possibility that the values of $q_{i}$ and $\boldsymbol{\eta}_{i}$ will be randomized using (8) and (10), respectively, which can 
induce both explorations and exploitations on $\boldsymbol{x}_{\boldsymbol{i}}$ throughout the entire optimization procedure. Experimental results (Tables 2-7) clearly show that $\mathrm{ABC}-\mathrm{AX}^{2}$ has significantly improved its results over the basic $\mathrm{ABC}$ algorithm [2] as well as several other recent variants of $\mathrm{ABC}$ (e.g., [15]-[21]).

There may be several possible future research directions based on this study. Firstly, $A B C-A X^{2}$ uses a simple strategy to adjust the control parameters $-p_{i}, q_{i}$ and $\boldsymbol{\eta}_{i}$ for each candidate solution $\boldsymbol{x}_{\boldsymbol{i}}$. A more sophisticated strategy, such as considering the properties of fitness landscape around $\boldsymbol{x}_{\boldsymbol{i}}$, or using a strategy parameterized by existing population diversity or the maturity of the optimization process may be more effective to balance between exploitations and explorations around $\boldsymbol{x}_{i}$. Secondly, the quality of the final solution could be improved further by using an exploitative and efficient local searcher. This may pinpoint the global minimum more precisely. Thirdly, $\mathrm{ABC}-\mathrm{AX}^{2}$ can be hybridized with many other existing evolutionary, swarm intelligence, machine learning techniques to further improve its results. Finally, $A B C-A X^{2}$ has been employed on the continuous problems. It would be interesting to know how well $\mathrm{ABC}-\mathrm{AX}^{2}$ performs on other existing problems, especially the discrete and real world ones.

\section{REFERENCES}

[1] D. Karaboga, "An idea based on honey bee swarm for numerical optimization", Erciyes University, Kayseri, Turkey, Technical Report-TR06, 2005.

[2] D. Karaboga, B. Akay, "A comparative study of artificial bee colony algorithm", Applied Mathematics and Computation 214 (1) (2009) 108-132.

[3] Q. Bai, X. Yun, "A new hybrid artificial bee colony algorithm for the traveling salesman problem", in: Proc. 3rd Int. Conf. Communication Software and Networks (ICCSN), 2011, pp. 155-159.

[4] N. Stanarevic, M. Tuba, N. Bacanin, "Modified artificial bee colony algorithm for constrained problems optimization", Int. Journal of Mathematical Models and Methods in Applied Sciences 5 (3) (2011) 644-651.

[5] S. Omkar, J. Senthilnath, R. Khandelwal, G. Naik, S. Gopalakrishnan, "Artificial bee colony (ABC) for multiobjective design optimization of composite structures", Applied Soft Computing 11 (1) (2011) 489-499.

[6] F. Kang, J. Li, Q. Xu, "Structural inverse analysis by hybrid simplex artificial bee colony algorithms", Computers and Structures 87 (13-14) (2009) 861-870.

[7] R. Irani, R. Nasimi, “Application of artificial bee colonybased neural network in bottom hole pressure prediction in underbalanced drilling", Journal of Petroleum Science and Engineering 78 (1) (2011) 6-12.

[8] N. Karaboga, "A new design method based on artificial bee colony algorithm for digital IIR filters", Journal of the Franklin Institute 346 (4) (2009) 328-348.

[9] D. Karaboga, B. Akay, "PID controller design by using artificial bee colony, harmony search and bees algorithms", in: Proceedings of the Institution of Mechanical Engineers, Part I: Journal of Systems and Control Engineering 224 (7) (2010) 869-883.

[10] R. Rao, P. Pawar, "Parameter optimization of a multi-pass milling process using non-traditional optimization algorithms", Applied Soft Computing 10 (2) (2010) 445-456.
[11] D. Karaboga, B. Gorkemli, C. Ozturk, N. Karaboga, “A comprehensive survey: artificial bee colony (ABC) algorithm and applications", Artificial Intelligence Review (2012) 1-37.

[12] L. Bao, J. Zeng, "Comparison and analysis of the selection mechanism in the artificial bee colony algorithm", in: Proc. 9th Int. Conf. Hybrid Intelligent Systems, 2009, pp. 411-416.

[13] W. Gao, S. Liu, "A modified artificial bee colony algorithm", Computers and Operations Research 39 (3) (2012) 687-697.

[14] J. Lampinen, I. Zelinka, "On stagnation of the differential evolution algorithm", in: Proc. 6th Int. Mendel Conf. on Soft Computing, 2000, pp. 76-83.

[15] M. S. Alam, M. M. Islam, "Artificial bee colony algorithm with self-adaptive mutation: A novel approach for numeric optimization", in: Proc. 2011 IEEE Int. Conf. on Trends and Developments in Converging Technology (TENCON), 2011, pp. 49-53.

[16] M. Abd, "A cooperative approach to the artificial bee colony algorithm", in: IEEE Congress on Evolutionary Computation (CEC), 2010, pp. 1-5.

[17] W. Lee, W. Cai, "A novel artificial bee colony algorithm with diversity strategy”, in: Proc. 7th Int. Conf. Natural Computation, 2011, pp. 1441-1444.

[18] B. Wu, S. Fan, "Improved Artificial Bee Colony Algorithm with Chaos", in: Y. Yu, Z. Yu, J. Zhao (Eds.): Computer Science for Environmental Engineering and EcoInformatics, Part I, Communications in Computer and Information Science, vol. 158, 2011, pp. 51-56.

[19] L. Fenglei, D. Haijun, F. Xing, "The parameter improvement of bee colony algorithm in TSP problem", Science Paper Online, November 2007.

[20] G. Zhu, S. Kwong, "Gbest-guided artificial bee colony algorithm for numerical function optimization", Applied Mathematics \& Computation 217 (7) (2010) 3166-3173.

[21] F. Kang, J. Li, Z. Ma, H. Li, "Artificial bee colony algorithm with local search for numerical optimization", Journal of Software 6 (3) (2011) 490-497.

[22] F. Qingxian, D. Haijun, "Bee colony algorithm for the function optimization", Science Paper Online, 2008.

[23] H. Quan, X. Shi, "On the analysis of performance of the improved $\mathrm{ABC}$ algorithm", in: 4th IEEE Int. Conf. Natural Computation (ICNC), 2008, pp. 654-658.

[24] E. Montes, R. Koeppel, "Elitist artificial bee colony for constrained real-parameter optimization", IEEE Congress on Evolutionary Computation 11 (2010) 1-8.

[25] S. Nieberg, H. Beyer, "Self-adaptation in evolutionary algorithms", Parameter Setting in Evolutionary Algorithm (2007) 47-76.

[26] J. Liang, A. Qin, P. Suganthan, S. Baskar, "Comprehensive learning particle swarm optimizer for global optimization of multimodal functions", IEEE Trans. on Evolutionary Comput. 10 (3) (2006) 281-295.

[27] C. Lee, X. Yao, "Evolutionary programming using mutations based on the Lévy probability distribution", IEEE Transactions on Evolutionary Computation 8 (1) (2004) 1-13.

[28] X. Yao, Y. Liu, G. Lin, "Evolutionary programming made faster", IEEE Transactions on Evolutionary Computation 3 (2) (1999) 82-102. 